\title{
ESTUDIO DE CORPUS: ESTRUCTURA Y LEGIBILIDAD EN EL DOCUMENTO DE CONSENTIMIENTO INFORMADO EN EL ÁMBITO ACADÉMICO-PROFESIONAL DE LAS CIENCIAS BIOMÉDICAS ${ }^{1}$
}

\author{
CORPUS STUDY: THE STRUCTURE AND LEGIBILITY IN THE \\ INFORMED CONSENT DOCUMENTS IN THE ACADEMIC- \\ PROFESSIONAL SCOPE OF THE BIOMEDICAL SCIENCES
}

\author{
ADRIANA RIBEIRO-ALVES \\ Universidad de Concepción. \\ adriribeiro@udec.cl \\ ANITA FERREIRA CABRERA \\ Universidad de Concepción. \\ aferreir@udec.cl
}

\section{RESUMEN}

Tradicionalmente, los investigadores obtienen un consentimiento de parte de sujetos participantes con documentos que detallan las características del estudio que se pretende realizar, aclarando los riesgos y beneficios que esto comporta, tanto para el sujeto de estudio como para el avance de la disciplina en cuestión, así como de la voluntariedad de la participación, brindando así también, la información de contacto tanto del investigador principal o responsable como del respectivo comité que conoce del estudio. En la revisión de la literatura producida en Chile respecto al documento de Consentimiento Informado, se observa un considerable número de textos escritos de diversa índole, principalmente de distintas especialidades de las ciencias biomédicas, sin embargo, no se ha estudiado este género como parte del discurso especializado en el ámbito académico-profesional. El estudio que aquí se presenta tiene como objetivos identificar la estructura básica del documento de Consentimiento Informado y evaluar la legibilidad de sus textos. Para ello se analizaron

\footnotetext{
${ }^{1}$ Este estudio forma parte de la tesis doctoral "La caracterización lingüística del género consentimiento informado en investigaciones científicas en el ámbito biomédico" de la autora principal, Adriana Ribeiro-Alves, dirigida por la Dra. Anita Ferreira Cabrera. Este estudio de corpus se basa en el modelo metodológico desarrollado en el contexto del proyecto de investigación FONDECYT $\mathrm{N}^{\circ} 1180974$ "Diseño e implementación de un corpus escrito de aprendientes de ELE en formato computacional para el análisis de la interlengua", cuya investigadora responsable es la Dra. Anita Ferreira Cabrera.
} 
los aspectos estructurales de un corpus textual compuesto por 176 documentos de consentimiento informado y se estableció el índice de legibilidad de dichos documentos, caracterizando así un problema desde distintas perspectivas: lingüística bioética y bioderecho.

Palabras clave: Corpus, legibilidad, consentimiento informado, ciencias biomédicas, discurso académico-profesional.

\section{ABSTRACT}

Traditionally, researchers obtain a consent from participating subjects with documents that detail the characteristics of the study that is intended, clarifying the risks and benefits that this entails, both for the subject of study and for the advancement of the related discipline, as well as the voluntariness of the participation, thus also providing the contact information of both the principal or responsible investigator and the respective committee that knows the study. In the review of the literature produced in Chile regarding the document of Informed Consent, we observed a considerable number of written texts of various kinds, mainly from different specialities of the biomedical sciences, however, this genre has not been studied as part of the discourse specialized in the academic-professional field. The main goals of the study presented here is to identify the basic structure of the Informed Consent document and to evaluate the legibility of these texts. In this research, the structural aspects of a textual corpus composed of 176 documents of informed consent were analyzed, establishing that these documents have a level of readability and low comprehension, thus characterizing a problem of approach both from the point of view of linguistics as much as to Bioethics or Biolaw.

Keywords: Corpus, legibility, informed consent, biomedical sciences, academic-professional discourse.

Recibido: 10.08.2018. Aceptado: 14.11.2018.

\section{INTRODUCCIÓN}

L as investigaciones científicas que involucran seres humanos, pese a su necesidad para el avance de la ciencia, algunas veces pueden resultar un problema para los investigadores. Cuando los objetivos de la investigación que tienen como propósito realizar contribuciones especialmente en el ámbito de la salud humana, se justifica involucrar seres humanos como parte condicionante para su desarrollo, haciendo que los investigadores puedan considerar que los resultados de sus estudios sean más importantes que proveer información y protección a los sujetos participantes.

En Chile, la Ley N²0.120 del año 2006, que trata "sobre la investigación cien- 
tifica en el ser humano, su genoma, y prohibe la clonación humana”, establece garantías y obligaciones en torno a la comunicación que debe existir desde comunidades discursivas especializadas, científicas y/o académicas, orientado a una audiencia no especializada, como son los sujetos participantes de los estudios. Este acto de comunicación debe cumplir con determinadas características para posibilitar la investigación científica que involucra seres humanos, las que deben considerar expresarse a través de un documento de Consentimiento Informado. Es con esta disposición, que consta en el párrafo segundo del Artículo 11 de la mencionada ley, que queda evidente el necesario aporte de la Lingüística Aplicada en el ámbito de la regulación jurídica de este documento, que se enmarca en la aplicación de principios bioéticos proporcionados como marco por la Declaración Universal sobre Bioética y Derechos Humanos (UNESCO, 2005), y la positivación de estos en el contexto de la investigación científica, disciplinando de esta forma la relación investigador-investigado o sujeto de investigación. Es por ello que el documento debe permitir la expresión de la autonomía por parte del sujeto de investigación, y esto está condicionado a que el texto escrito especializado cuente con un nivel de información adecuado, suficiente y comprensible, siempre formalizado en el acta escrita.

Se puede entender el documento de Consentimiento Informado, cuya necesidad es legal, como un texto en el cual se hace efectiva la entrega expresa de la información revestida de características determinadas, de parte del investigador hacia quien la recibe, quien es a su vez el sujeto de investigación. Este sujeto debe entender y analizar dicha información para deliberar y decidir, aceptando o rechazando, o hasta desistiendo de su aceptación en participar de la investigación científica. De ese modo, existe una proposición condicionante en el sentido de que el éxito de la investigación científica depende del uso del lenguaje que valida el diálogo entre las partes involucradas en la dinámica del proceso de Consentimiento Informado, es decir, por un lado hay una elaboración y confección del texto del discurso especializado académico-profesional y, por otro, el entendimiento de éste por el sujeto investigado, no conocedor del léxico especializado considerado en el documento en estudio.

Si bien en el ámbito académico chileno, los estudios sobre documentos de Consentimiento Informado han estado incrementándose en el tiempo (De la Maza Gazmuri, 2017; Kottow, 2016; Figueroa, 2012; Lolas Stepke, 2010; Santos, 2008), aún es incipiente el estudio de este género como texto del discurso especializado en el área de la Lingüística de Corpus. En efecto, en el contexto actual en el ámbito nacional son escasos los estudios que consideran un análisis con rigor científico desde los estudios de Corpus, como también respecto a los estudios de este texto especializado adoptado ya sea en las ciencias biomédicas o ciencias sociales.

En la revisión de la literatura producida en Chile respecto al documento de Consentimiento Informado, se observa un considerable número de textos escritos 
de diversa índole, principalmente de distintas especialidades de las ciencias biomédicas, desde la vigencia de la Ley $\mathrm{N}^{\circ} 20.584$, del año 2012, que "regula los derechos $y$ deberes que tienen las personas en relación con acciones vinculadas a su atención en salud', y que en su Artículo 14 reconoce expresamente la garantía de aplicación de este documento. Sin embargo, no se ha estudiado este género como parte del discurso especializado en el ámbito académico-profesional. De dicha revisión se destaca referencias al documento en estudios relacionados a la Bioética, la que es reconocida como disciplina y es parte de planes de estudio de cursos superiores en el ámbito de las ciencias biomédicas, llegando incluso a ser parte de una Comisión de alto nivel en el Ministerio de Salud de Chile (2011) y que, por otra parte, el Bioderecho busca dar un tratamiento de acuerdo de voluntades.

Saber producir, organizar y escribir el Consentimiento Informado, cuya estructura es, como su propio nombre dice, una parte informativa y otra permisiva, condescendiente (otorgativa o limitativa), de modo que siempre sea consentida (Austin, 1982 y Searle, 1986), constituye una nueva área para el estudio de la Lingüística Aplicada en Chile, en el ámbito de la Lingüística de Corpus y del género del discurso académico-profesional. En consecuencia, el estudio que aquí se presenta tiene como principal propósito tratar de identificar la estructura básica y la legibilidad del documento de Consentimiento Informado, de modo que resulte coherente con lo que dispone la Declaración Universal sobre Bioética y Derechos Humanos (UNESCO, 2005), que se resume en:

- orientar el proceso de información y de debate sobre la investigación científica o la medida diagnóstica o terapéutica propuesta entre el investigador responsable y el sujeto de la investigación (o el prestador de salud y el paciente);

- servir de registro de que ese proceso se ha llevado a cabo según lo dispuesto en las leyes y,

- garantizar la protección legal de respeto a la autonomía del sujeto que participa como voluntario en la investigación científica (o del paciente).

Para dar cuenta de estas funciones, los documentos de Consentimiento Informado deben contener párrafos específicos con la información acerca de la investigación científica que se pretende ejecutar, contemplando la participación del sujeto -ser humano- y también, de la declaración de éste en involucrarse en el estudio mediante su participación libre, con la expresa manifestación de voluntad. De ese modo, este documento garantiza que el sujeto no sólo accede a participar sino también autoriza la aplicación de procedimientos de estudios que colaboran con el desarrollo del conocimiento científico.

A partir de estas constataciones y considerando la necesidad de la verdadera transparencia y validez en la comunicación, este estudio hace su contribución en el ámbito del discurso académico-profesional a través de la descripción detallada 
de la superestructura del género discursivo "consentimiento informado" del área biomédica y de los niveles de legibilidad que se pueden observar en un corpus de dicha área. Para ello se buscó caracterizar la forma y la naturaleza de los textos y delimitar el grado de legibilidad de la información que contienen dichos documentos para ser comprendidos.

\section{MARCO DE REFERENCIA}

El término "Consentimiento Informado", según el Diccionario de la Lengua Española de la Real Academia de la Lengua Española (2018), se define como:

1. m. Der. consentimiento que ha de prestar el enfermo o, de resultarle imposible, sus allegados, antes de iniciarse un tratamiento médico o quirúrgico, tras la información que debe transmitirle el médico de las razones y riesgos de dicho tratamiento.

Con esta definición que ubica a una acción de "prestar" como comunicar, el Consentimiento Informado se instrumentaliza, inicialmente, en un proceso dialógico en el que una persona acepta participar en un tratamiento médico o quirúrgico, conociendo sus razones y los riesgos que se puedan presentar durante el desarrollo de la aplicación propia de la Medicina. Aunque de esta manera lo hace acotado a un área del conocimiento (Medicina) y apegada a su antecedente histórico, la definición debe ser adaptada al contexto de la especificidad de cada área del conocimiento. Por lo tanto, se puede argüir, desde la perspectiva lingüística y de manera innovadora, que el Consentimiento Informado es una comunicación que se caracteriza necesariamente por su estructura dialógica, la que está contemplada para todas las áreas del conocimiento que involucren por un lado un académico o profesional, en su rol de investigador científico, y por otro el sujeto de investigación, un ser humano con personalidad física, cuya finalidad es informar las razones de sus roles en el estudio y respectivas protecciones, todo caracterizado en un texto escrito para un contexto específico.

También se caracteriza por ser el instrumento de institucionalización del discurso ético en el campo de la investigación científica (López Calera, 2000) en clara referencia a la exigencia de la comprensión de la información por parte del sujeto del estudio, considerado como una persona con derechos y deberes y no sólo como una muestra de un universo que está contemplado para la inclusión en una investigación, lo que sitúa el saber que consiente, a través de un procedimiento formal.

En los últimos años se ha intensificado el interés por el estudio de la variación lingüística a través del discurso en las disciplinas científicas (Parodi, 2007a). Esta 
particular orientación se constituye como un factor preponderante en la articulación de un conocimiento y léxico especializado al interior de una comunidad discursiva como la científica académica. Hyland (2000) establece que no existe tal discurso disciplinario especializado y de que se hace más relevante referir a discursos disciplinarios en forma general, siendo éste un hecho cada vez más reconocido entre los investigadores (Bhatia, 2004).

Desde una perspectiva de los géneros discursivos, el Consentimiento Informado es un texto por el cual se entrega información precisa, con determinadas características, desde el investigador hacia el sujeto de investigación y/o paciente, quien comprendiendo y analizando dicha información, pasa a deliberar y decidir, aceptando o rechazando, o hasta desistiendo de su aceptación inicial, en participar de la investigación científica o de un tratamiento médico. Además, la normativa nacional, que va en línea con la Declaración Universal sobre Bioética y Derechos Humanos (UNESCO, 2005), garantiza que la validación científica no sólo depende de la metodología que se describe para aplicar un estudio científico, sino también, de un documento de Consentimiento Informado que se traduce en la libre manifestación de voluntad, previa y expresa, para el cual se informa al sujeto de estudio acerca de su participación en él. De ese modo, las normas anteriormente indicadas dejan explícito que el éxito de la investigación científica o del cuidado o tratamiento médico depende del uso del lenguaje que se logra al dialogar las partes involucradas en la dinámica del proceso de Consentimiento Informado, que por un lado se realiza con la elaboración y confección del texto del discurso especializado académico-profesional y, por otro, con la verdadera comprensión de éste por el sujeto investigado y/o beneficiario de la prestación en salud.

La comprensión del modo en que el conocimiento disciplinar (discurso especializado) se construye y se valora en contextos científico-académicos y profesionales, debe su avance al enfoque que proporciona la lingüística de corpus, posibilitando análisis de rasgos lingüísticos, sus funciones y distribuciones en el discurso natural, en este caso de estudio, en contextos del área biomédica, a través de los consentimientos informados (Parodi y Ferrari, 2007).

Los primeros estudios acerca de los análisis de legibilidad lingüística en el ámbito de las ciencias de la salud han sido desarrollados desde los años 70 en los Estados Unidos (Pyrczak y Roth, 1976; Liguori, 1978) y de los 90 en España (Simón-Lorda y Concheiro-Carro, 1993; Simón-Lorda y Barrio-Cantalejo, 1995; Barrio-Cantalejo y Simón-Lorda, 2003). Esta perspectiva en la investigación estaría contribuyendo en los estudios de Consentimiento Informado, específicamente en lo relacionado con la característica referida a la autonomía del sujeto de investigación/paciente. De ese modo, la autonomía estaría reflejada en la legibilidad de un texto escrito, cuya información entregada debería ser adecuada y suficiente. Así también se encuentran estudios más recientes acerca de la legibilidad de documentos de Consentimiento Informado escritos en inglés o en la lengua castellana, 
los cuales confirman el interés por esta línea de investigación en el ámbito de la legibilidad de dicho documento en el ámbito de las ciencias biomédicas (Ramírez Durán, 2017; Ramírez-Puerta, Fernández-Fernández, Frías-Pareja, Yuste-Ossorio, Narbona Galdó y Peñas-Maldonado, 2013).

Hasta la fecha, la revisión bibliográfica sobre el documento de Consentimiento Informado evidencia que dicho tipo de discurso, en general, se ha tratado como necesario para ejecutar una investigación científica (Emmanuel, 1999), o como un documento ético-jurídico o tan sólo jurídico que busca la protección legal de sujetos de derecho en una investigación (Bonilla-Sandi, 2003; Jiménez-Sánchez, Lara, Arellano-Méndez. 2010) y además, como un documento médico-jurídico en las investigaciones o intervenciones biomédicas (Ley No 20.120, de 2006 y Ley $\mathrm{N}^{\circ}$ 20.584, de 2012). Sin embargo, se hace necesario establecer este texto como un género discursivo que documenta decisiones, procesos de elección y combinación (De Beaugrande y Dressler, 2005), materializándolo como un objeto del estudio de la Lingüística Aplicada, desde su perspectiva multidisciplinaria, atendiendo a su uso en los contextos académico-profesionales, y contribuyendo en su descripción en el contexto Bioético y en el Bioderecho (Emmanuel, 1999).

$\mathrm{La}$ exigencia expresa de un Consentimiento Informado según la Ley $\mathrm{N}^{\circ}$ 20.120, del año 2006, cuando dispone que "Toda investigación científica en un ser humano deberá contar con su consentimiento previo, expreso, libre e informado, o, en su defecto, el de aquel que deba suplir su voluntad en conformidad con la ley", revela un derecho que tiene el sujeto de investigación de otorgar o denegar de forma libre su voluntad en participar de una investigación científica biomédica, siempre condicionada a la entrega de información adecuada, suficiente y comprensible, firmando al final del texto del documento. Sin embargo, la ley y su reglamento, Decreto $N^{\circ} 114$ del Ministerio de Salud, Subsecretaría de la Salud Pública, publicado en 2011, no especifica que se debe entender por información adecuada, suficiente y comprensible, permitiendo al investigador producir, organizar y escribir un texto que él estime que reúna estos requisitos. Esta situación podría obviar que la información adecuada, suficiente y comprensible es hacia al sujeto participante en la investigación, lo que podría ser establecido a través de índices de legibilidad. Es así como un estudio sobre el conjunto de características lingüísticas de este tipo de texto permitiría leerlo y comprenderlo con mayor facilidad (Alliende González, 2002).

Consecuente con lo antes expuesto, este estudio que se presenta considera una recolección y análisis de un corpus de documentos de Consentimiento Informado, aplicados en contextos académico-profesionales, cuya estructura y caracterización será determinada con el reconocimiento del texto escrito especializado como un género del discurso y por su propósito comunicativo, que es la información, como garantía de protección del sujeto de investigación científica. 


\section{METODOLOGÍA}

El estudio que se presenta busca caracterizar la forma y la naturaleza del texto y delimitar el grado de legibilidad de la información que contienen los documentos de Consentimiento Informado para ser comprendidos. El diseño de la investigación realizada corresponde, así, a un estudio descriptivo de corte transversal con un enfoque de análisis de datos mixto de tipo cuantitativo y cualitativo.

Se recolectaron 176 (ciento setenta y seis) textos de documentos de Consentimiento Informado, escritos en idioma español, pertenecientes al ámbito académico-profesional y relacionados con el área disciplinar de las ciencias biomédicas. El periodo de recolección textual comprende desde enero de 2014 hasta el primer semestre de 2018. Todos los documentos recolectados contaban con la revisión de Comités de Ética para ser aplicados por investigadores a sujetos de estudio, voluntarios participantes, de investigaciones científicas. Dichos documentos provenían tanto de universidades chilenas como de instituciones públicas y privadas que realizan actividades de investigación en Chile y se presentaron en condición de modelo a ser seguido o pauta que se aconseja utilizar. Además, cabe destacar aquí que el corpus está constituido por documentos de consentimiento destinados a personas mayores de 18 años de edad, capaces civilmente.

Se utilizó la técnica de análisis de contenido para identificar presencia o ausencia de categorías necesarias y deseables, y se estableció dificultades en la comprensión del lenguaje en los formatos de dos categorías: 1) lenguaje cotidiano (común) y 2) lenguaje especializado o científico, específico del léxico académicoprofesional.

La investigación se desarrolló bajo un enfoque metodológico mixto, por cuanto es el sugerido para el análisis de discurso especializado, orientado por la teoría Top-down approaches, como son las estrategias de procesamiento de información con el uso de software que considera evaluar los modelos de los documentos desde una mirada de información global, hasta ir abordando variables más detalladas y específicas, conforme a lo delimitado por Biber, Connor y Upton (2007) y Parodi (2002, 2003), basados en la propuesta original de Swales (2004). Primero se buscó recolectar datos cualitativos y, después, los cuantitativos, dado que esta secuencia permitiría explorar el planteamiento del problema para expandir su entendimiento en una muestra robusta, generada en un estudio piloto.

Como ya se ha señalado, la propuesta de este estudio fue delimitar los rasgos o características más relevantes y frecuentes en los documentos de Consentimiento Informado, analizando la estructura del documento y el índice de legibilidad de estos a partir del índice de legibilidad INFLESZ.

Con estos elementos se estableció el propósito comunicativo del documento, en términos de la información al sujeto y su declaración de aceptación en participar de una investigación científica en el ámbito de las ciencias biomédicas. 


\section{PROCEDIMIENTOS}

La totalidad de los textos se obtuvieron digitalizados en formato .pdf o .doc y fueron convertidos a texto plano .txt para ser compatibles con la aplicación de los instrumentos de análisis ejecutados en este estudio.

La construcción del corpus consideró los siguientes aspectos relevantes (Parodi, 2007b): extensión, formato, representatividad, diversificación, marcado o etiquetado, procedencia, tamaño de las muestras, clasificación y adscripciones de tipos disciplinar.

La extensión promedio de los documentos que hicieron parte del corpus fue de dos páginas en promedio. Se estableció posteriormente un formato dividido en partes integrales, que se pudo advertir en al menos un $40 \%$ de los textos. En estos documentos fueron corregidos los errores derivados de la conversión del formato y excluidos los datos de los centros de investigación y nombres de los investigadores (investigador responsable, investigador principal y, si fuera el caso, de los co-investigadores) (ver Anexo). Posteriormente fueron agrupados y etiquetados conforme su procedencia del ámbito académico-profesional (investigación) en las ciencias biomédicas.

\subsection{Lineamientos de análisis}

Construida la base de datos, se llevó a cabo el procesamiento de los datos, atendiendo a los siguientes aspectos o rasgos:

1. a las estructuras de los documentos recolectados,

2. al léxico especializado y análisis de frecuencia de palabras,

3. al contexto de uso del lenguaje especializado, con analisis de concordancia,

4. a la delimitación del indice de legibilidad de los textos del corpus.

Se evaluaron estos aspectos mediante el índice de legibilidad INFLESZ, el cual mide la facilidad o la dificultad de leer un texto y ha sido desarrollado y validado para su uso como indicador de la dificultad de textos escritos en la lengua castellana. Técnicamente, el cálculo de este atributo (índice) considera el número total de sílabas por palabra, número de palabras y se relaciona al número de frases en el texto (Barrio-Cantalejo, Simón-Lorda, March Cerdá y Prieto Rodríguez, 2008):

$$
I=206.835-\frac{62.3 S}{P}-\frac{P}{F}
$$

donde $\mathrm{S}$ corresponde al total de sílabas; $\mathrm{P}$, la cantidad de palabras y $\mathrm{F}$, el número de frases. 
En este contexto, se entiende por palabras (P), las unidades de la lengua dotadas de significado y sílabas $(\mathrm{S})$, como un grupo de sonidos que se pronuncia en una sola emisión de voz. En relación con las frases $(\mathrm{F})$, se reconoce como la palabra o conjunto de palabras separados por signos tales como dos puntos, punto y coma, punto gramatical/final, guión, de interrogación y de exclamación.

La escala de legibilidad INFLESZ es frecuentemente usada para evaluar la legibilidad de documentos de consentimiento informado, informes y materiales para la educación en el ámbito de la salud (Barrio-Cantalejo, 2015).

Para obtener este índice se empleó como herramienta el software Legible (http://legible.es), accesible en la plataforma web y adaptada al lector del idioma español.

Con la finalidad de evaluar la legibilidad de un texto y su relación con la comprensión, se emplea la fórmula de Lecturabilidad, herramienta basada en la prueba de legibilidad de Flesch, adaptada al español por Fernández-Huerta (1959), según la fórmula:

$$
L=206,84-0,60 P-1,02 F
$$

siendo $L$ la lecturabilidad; $P$ el promedio del número total de sílabas por cada 100 palabras y $F$ el promedio del número total de frases por cada 100 palabras. Este índice, también está disponible a través del programa Legible.

Para el análisis de frecuencia y extracción de concordancias se empleó el software AntConc. Para ello se consideró el uso del programa en su versión para Macintosh, versión 3.5.7. (23 de abril de 2018), describiéndose como un software libre para el análisis de corpus textuales desarrollado por el Dr. Laurence Anthony (2013), profesor en la Faculty of Science and Engineering, Waseda University, Japón.

\section{RESULTADOS}

\subsection{Estructura formal de los textos de los documentos de consentimiento informado}

Inicialmente, se consideró el análisis de la estructura formal de los textos. Para ello, se observó y registró los distintos apartados de las muestras de textos escritos de los documentos.

El propósito fue conocer el tipo de información escrita que incluye, cómo se distribuye dicha información y si existe un patrón más o menos establecido de documento de Consentimiento Informado como género textual desde su estructura formal.

En este caso, se procedió de forma manual con la toma de datos, respecto de 
cada uno de los documentos, a fin de registrar las características de las secciones o apartados que lo componen.

Se constató el registro de las secciones de cada uno de los 176 (ciento setenta y seis) textos de los modelos de documento de Consentimiento Informado, atendiendo a los siguientes aspectos:

1. El encabezado del texto, en el cual se registra el uso de logotipo(s) institucional(es) en un $43,7 \%$ de los casos, el título del documento que resultó ser predominante como "Consentimiento Informado", en un $36 \%$, seguido de "Documento de Consentimiento Informado", con un $16 \%$. La Tabla I muestra los detalles del análisis de los títulos del documento.

Tabla I. Encabezamiento o título de los documentos analizados.

\begin{tabular}{|l|c|}
\hline \multicolumn{1}{|c|}{ Título del documento } & \% frecuencia \\
\hline Consentimiento Informado & 36 \\
\hline Documento de Consentimiento Informado & 16 \\
\hline Formulario de Consentimiento Informado & 15 \\
\hline Información y Formulario de Consentimiento Informado & 7 \\
\hline Acta de Consentimiento Informado & 5 \\
\hline Declaración de Consentimiento Informado & 4 \\
\hline Formulario de Consentimiento e Información & 3 \\
\hline $\begin{array}{l}\text { Información para el Participante y Documento de Consentimiento } \\
\text { Informado }\end{array}$ & 3 \\
\hline Información para el Participante y Consentimiento de Participación & 2 \\
\hline Información al Participante & 2 \\
\hline Información y Consentimiento & 2 \\
\hline Sin título & 2 \\
\hline $\begin{array}{l}\text { Formulario Genérico de Información al Participante y Consentimiento } \\
\text { Informado }\end{array}$ & 1 \\
\hline Ficha Informativa para participar en Estudio de Investigación & 1 \\
\hline Protocolo de Investigación & 1 \\
\hline
\end{tabular}

2. La información, que se identifica como primera parte del texto del documento de Consentimiento Informado, que se visualiza como:

2.1. general, cuando corresponde al título del estudio e identificación institu- 
cional del/de los Investigador(es), responsable(s) o principal y co-investigadores, vinculando sus nombres a la unidad(es) ejecutora(s) del estudio, y 2.2. especifica, cuando se trata de la información referida a: (a) el objetivo del estudio, (b) la fundamentación de la participación, libre y voluntaria del sujeto en la investigación, (c) los procedimientos propios de la intervención, (d) los posibles beneficios y riesgos, precisándose cómo será mitigada o gestionada, (e) la confidencialidad, (f) la compensación económica, (g) las garantías de conocer los resultados del estudio, (h) los derechos a retirarse o no aceptar participar, y finalmente, (i) la información referida a el o los contacto(s) con el/ los investigadores responsable(s) o principal del proceso de Consentimiento Informado.

3. La declaración del consentimiento, en que el sujeto declara su entendimiento, después de haber leído el documento, y acepta a participar, autorizando a que se proceda aplicar los procedimientos de intervención que propone el investigador responsable al sujeto de investigación, no reservándose de hacer preguntas ante dudas.

4. Finalmente, la validación de la legalidad del documento que se da con la fecha y las firmas de las partes involucradas en el estudio, siendo el participante, el investigador responsable o principal y el director del centro o establecimiento, donde se lleva a cabo la investigación, o su delegado, quien figura como ministro de fe.

En consecuencia, sobre un $40 \%$ de los documentos de Consentimiento Informado analizados en el corpus, que en promedio eran de 02 (dos) páginas, cuentan con un formato que puede ser dividido en 04 (cuatro) partes (ver Tabla II):

Tabla II. Formato de los documentos de Consentimiento informado del área biomédica.

\begin{tabular}{|c|c|}
\hline 1. & $\begin{array}{l}\text { Encabezamiento } \\
\text { a. logotipo institucional } \\
\text { b. título del documento }\end{array}$ \\
\hline 2. & $\begin{array}{l}\text { Información de la investigación científica. } \\
\text { a. general y } \\
\text { b. específica }\end{array}$ \\
\hline 3. & $\begin{array}{l}\text { Declaración: } \\
\text { a. de haber sido informado; } \\
\text { b. de haber leído y comprendido el documento con la información y } \\
\text { c. de aceptación y en participar como sujeto de investigación. }\end{array}$ \\
\hline
\end{tabular}


Continuación Tabla II.

\begin{tabular}{|c|l|}
\hline 4. & 4.1. Fecha y \\
4.2. & Firmas: \\
& a. del sujeto participante, \\
& b. del investigador responsable y \\
c. del director del centro de estudio o su delegado/ Ministro de Fe.
\end{tabular}

A partir de estos resultados se propone un modelo de estructura básica para este tipo de documento, como se puede observar en la Figura 1.

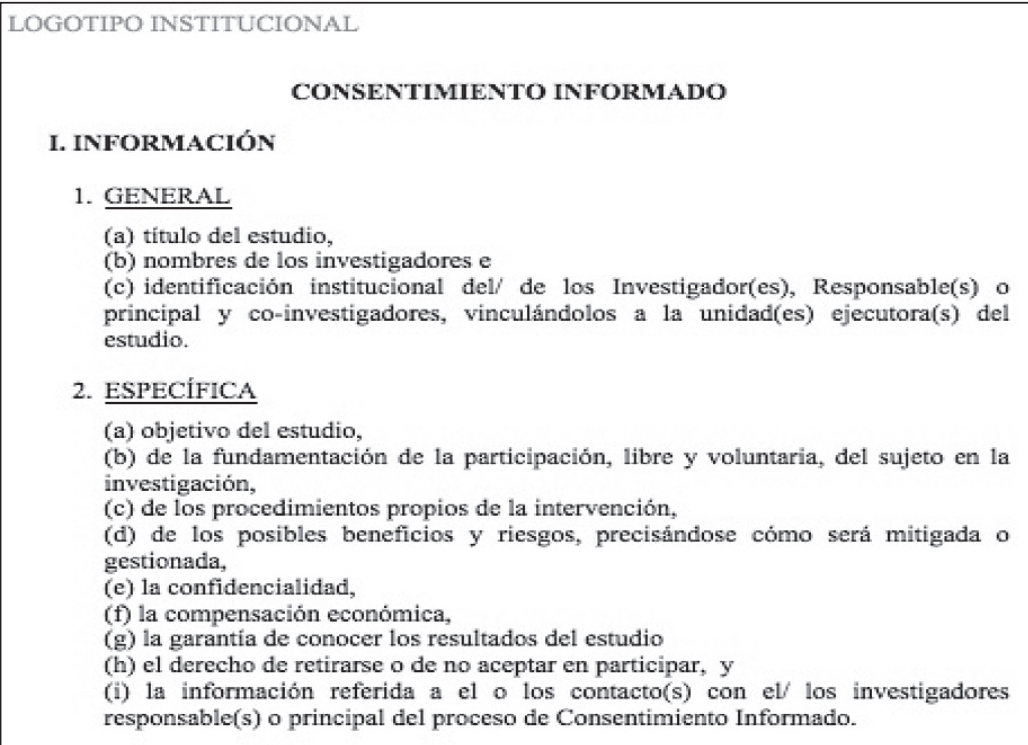

\section{INFORMACIÓN}

1. GENERAL
(a) título del estudio,
(b) nombres de los investigadores e
(c) identificación institucional del/ de los Investigador(es), Responsable(s) o principal y co-investigadores, vinculándolos a la unidad(es) ejecutora(s) del estudio.

\section{ESPECIFICA}
(a) objetivo del estudio,
(b) de la fundamentación de la participación, libre y voluntaria, del sujeto en la investigación,
(c) de los procedimientos propios de la intervención,
(d) de los posibles beneficios y riesgos, precisándose cómo será mitigada o gestionada,
(e) la confidencialidad,
(f) la compensación económica,
(g) la garantía de conocer los resultados del estudio
(h) el derecho de retirarse o de no aceptar en participar, y
(i) la información referida a el o los contacto(s) con el/ los investigadores responsable(s) o principal del proceso de Consentimiento Informado.

\section{DECLARACIÓN DE CONSENTIMIENTO}

(a) Constancia de que el sujeto de ha recibido la información adecuada y necesaria acerca de la investigación cientifica.

(b) Declaración de que el sujeto ha leido y entendido en su totalidad el documento.

(c) Declaración de que acepta a participar de manera libre y voluntaria en el estudio.

\section{Fecha}

Firma del Participante

Firma del Investigador Responsable

Firma del Director del Centro de Estudio o su delegado/ Ministro de Fe

Figura 1. Modelo de documento de Consentimiento Informado para ser aplicado en el ámbito de las ciencias biomédicas (Ribeiro-Alves y Ferreira, 2018). 


\subsection{Frecuencia de palabras}

El objetivo de este análisis fue determinar las palabras que con mayor frecuencia se encuentran en este tipo de documento, como se puede ver en la Tabla III.

Como criterios para la delimitación de las palabras más frecuentes con el uso de AntConc, se establecieron primero palabras repetidas 05 (cinco) o más veces, cuyos registros fueron de léxico no especializado en el ámbito de las ciencias biomédicas, pero sí de uso especializado en la lingüística jurídica y característico del acto de consentir (autorización) y, registro léxico de uso no especializado (común) y palabras repetidas 05 (cinco) o más veces, con registro de léxico especializado, pertenecientes a la investigación científica.

Estos datos permitieron observar que los léxicos guardan relación con la información y la declaración de la estructura formal presentada, excluyendo la palabra "Consentimiento", propio del documento de Consentimiento Informado, la que está en el encabezado y "firma", que es de uso no especializado, para la validación legal.

Tabla III. Palabras más frecuentes encontradas en el corpus, asociadas al formato del documento y a la investigación que se presenta, específicamente.

\begin{tabular}{|l|l|}
\hline \multicolumn{1}{|c|}{ Consentimiento Informado } & \multicolumn{1}{c|}{ Investigación } \\
\hline Estudio & Riesgos \\
\hline Investigación & Beneficios \\
\hline Información & Formulario \\
\hline Participar & Tratamiento \\
\hline Consentimiento & Procedimiento \\
\hline Investigador & Voluntaria \\
\hline Universidad & Desarrollo \\
\hline Participación & Confidencial \\
\hline Informado & Acuerdo \\
\hline Responsable & Evaluación \\
\hline $\begin{array}{l}\text { Además, se enumeran con frecuencia } \\
\text { nombres de investigadores o instituciones } \\
\text { académicas o centros de salud, incluidos } \\
\text { en las diferentes investigaciones. }\end{array}$ & $\begin{array}{l}\text { Se citan además, palabras que refieren nom- } \\
\text { bres específicos de medicamentos (nombre } \\
\text { químico o de fantasía y nombres de los } \\
\text { procedimientos o análisis que serán em- } \\
\text { pleados). }\end{array}$ \\
\hline
\end{tabular}




\subsection{Terminología especializada y extracción de concordancias}

El análisis se centró específicamente en la terminología especializada. Para ello se estableció ciertos límites entre un lenguaje de especialidad y la lengua común, considerando la transferencia léxica que se produce de la lengua general al lenguaje especializado, del lenguaje especializado a la lengua general o entre lenguajes de especialidad.

En el análisis de la estructura de los documentos se consideró la extracción de concordancias, en la que se buscó una palabra clave en el contexto del documento en estudio, con la aplicación de AntConc. Este análisis tuvo como propósito determinar el uso de los términos más frecuentes (ver Tabla III) en más de un contexto de uso en un mismo documento de consentimiento informado, asociado al formato del documento y al texto con el objetivo de la investigación, en el ámbito de las ciencias biomédicas.

En las Figuras 2 y 3 se aprecia tanto el aspecto referido a la formalidad del documento de Consentimiento Informado, como lo concerniente a la información específica que se entrega al sujeto de estudio. En efecto, a partir de los resultados que arroja AntConc sobre las concordancias de los términos "investigación" (Figura 2) y "riesgos" (Figura 3) se puede delimitar una clara relación fraseológica que limita su uso e interpretación inequívoca en el ámbito de las ciencias biomédicas, en consideración a lo que se realiza (investigación) y a lo que se considera como un aspecto inherente a esta investigación (riesgos). En ambos casos las palabras están relacionadas a léxico específico en relación a sus colocados, palabras ubicadas anterior o posteriormente al término cuya frecuencia se informa.

El grupo de palabras y frases que determina una terminología específica o léxico es de los elementos más importantes y evidentes en el análisis de la legibilidad y con ello, la comprensión de un documento de consentimiento informado, por lo que todo esfuerzo orientado a producir familiaridad con el sujeto de investigación se hace necesario, aunque con ello la extensión de estos textos se vea incrementada. 


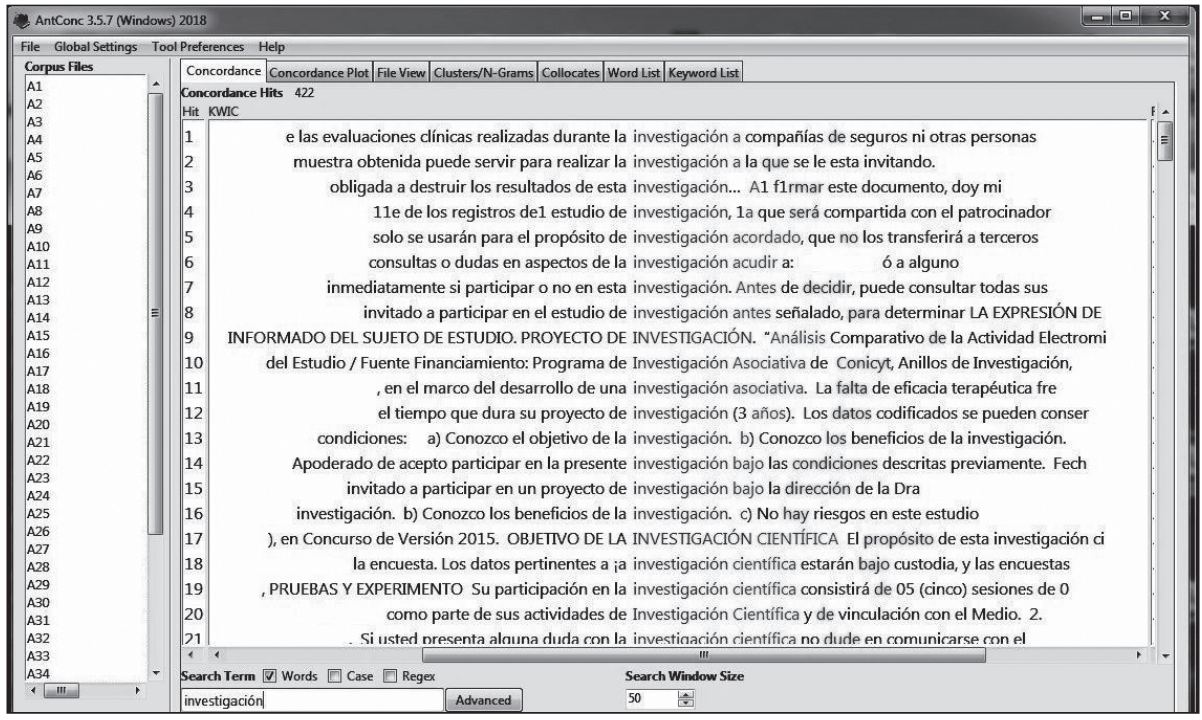

Figura 2. Concordancias asociadas a la palabra "investigación".

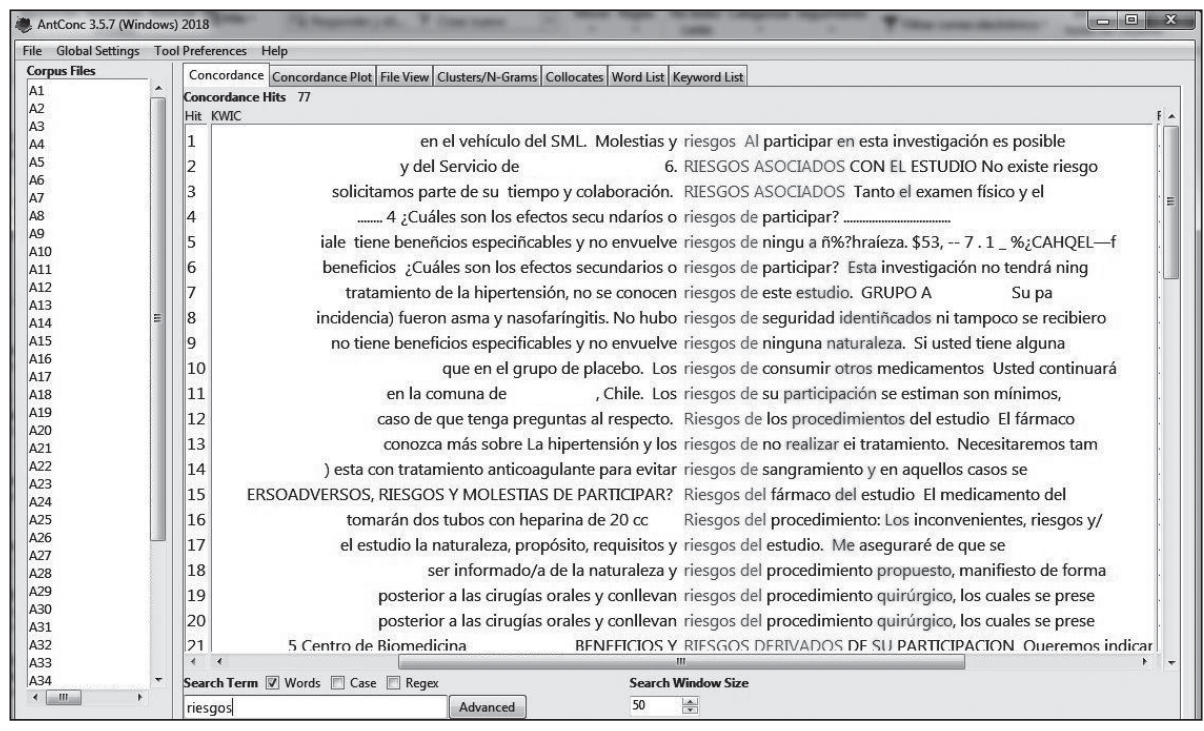

Figura 3. Concordancias asociadas a la palabra "riesgos". 


\subsection{Legibilidad}

La legibilidad es un índice obtenido a partir de un conjunto de parámetros (sílabas, cantidad de palabras y número de frases) que permite medir la complejidad del texto basado en características de las palabras o frases que forman parte del texto y la estructura del contenido en una pieza del enunciado.

La metodología para el uso de los sistemas de medición de legibilidad (Barrio-Cantalejo y Simón-Lorda, 2003; Ballesteros-Peña, Fernández-Aedo, 2013), sugiere que no se considere la primera parte del documento, siendo analizada la legibilidad de aquellas partes que contienen tanto la información del o los procedimiento(s) a realizar, así como de la declaración, a partir de la cual se obtendrá la anuencia o no de participar de la investigación científica o intervención en el ámbito de las ciencias biomédicas.

El análisis de los datos del corpus permite delimitar que la sección de la declaración está presente en un $78 \%$ de los documentos procesados, así como también la sección de la Información, en la cual se explicita el objetivo de la investigación, se presenta en un $100 \%$ de los textos del corpus.

Así también, en el corpus analizado, se evidenció documentos con un promedio de 132 párrafos de longitud y 1.293 palabras, en un rango entre 239 y 2.962 palabras totales, distribuidas en un promedio de 118,3 frases de 12,8 palabras de largo (promedio). La explicación de esta variabilidad en la longitud de los textos podría deberse a los documentos utilizados en la investigación, que corresponden a un modelo estándar con variantes de redacción que se condicen con el ámbito académico-profesional, como por ejemplo la falta de estructura de los textos, uso de abreviaturas, textos que inician con la declaración del participante sin antes entregar la información o incluso, no indicar el título del documento.

En cuanto a los niveles de legibilidad de la información obtenidos a partir del índice INFLESZ, se puede precisar que de los 176 documentos analizados, 128 textos $(72,8 \%)$ obtuvieron la clasificación de Algo Difícil de Leer y 48 (27,2\%) la categoría de Normal (veáse Gráfico 1). El índice INFLESZ que se obtiene en la plataforma Legible, permite delimitar que una parte importante de los textos no son fáciles de leer. Esta condición se puede atribuir al hecho de estar escritos con palabras de un vocabulario específico (véase Tabla II), las cuales resultan de difícil comprensión para los usuarios no especializados.

Similar apreciación se obtiene al aplicar el Indice de Fernández-Huerta (1959) de lecturabilidad o comprensión, donde 48 textos (27,3\%) se clasifican como Normal, 104 documentos $(59,1 \%)$ como Algo Difícil, y 24 textos $(13,6 \%)$ como Difícil (Gráfico 2).

La fluidez que los textos escritos ofrecen para ser comprendidos con bajo nivel de esfuerzo, es lo que se conoce como lecturabilidad. Considerando que dos textos similares pueden tener una categoría de más o menos fáciles de leer, dependiendo 
del lector, la lecturabilidad queda subordinada a distintos factores, como son la habilidad lectora, la formación y el conocimiento que posee el lector, entre otros. La lecturabilidad en un texto no supone que éste haya sido redactado usando un modo de expresión más familiar o de poca formalidad.

Dada la escasa comprensión de los textos del corpus, la cual se infiere del bajo grado de legibilidad o comprensión de los mismos, hacen aconsejable que se elaboren documentos de consentimiento informado que consideren el uso de un lenguaje menos especializado, de acuerdo a cada procedimiento o investigación, que permita alcanzar valores de legibilidad superiores al $70 \%$ para una mejor comprensión de los usuarios o sujetos muestrales.

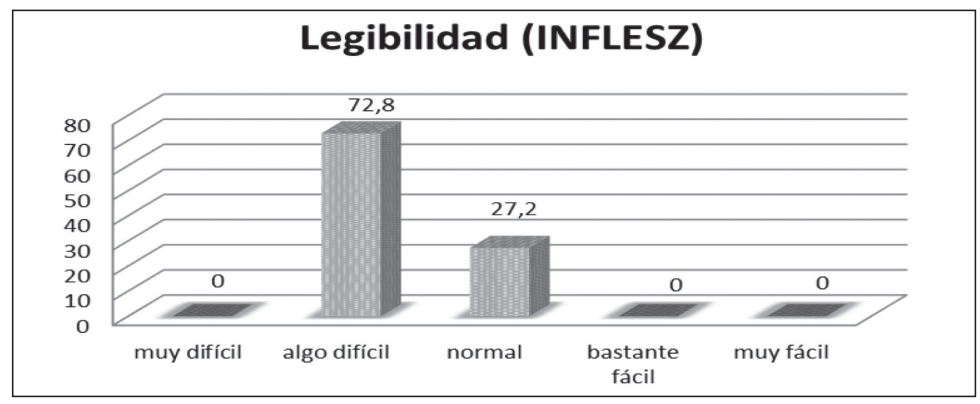

Gráfico 1. Legibilidad de los documentos del corpus de documentos de Consentimiento Informado del ámbito biomédico, analizados según cálculo INFLESZ (Barrio-Cantalejo, 2015).

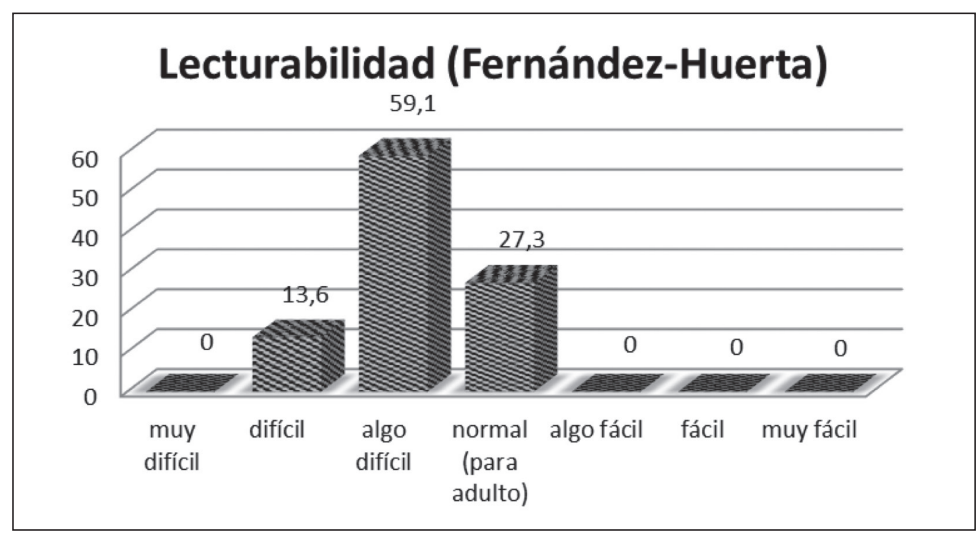

Gráfico 2. Lecturabilidad de los documentos del corpus de documentos de Consentimiento Informado del ámbito biomédico, analizados según cálculo (Fernández-Huerta, 1959). 
Lecturabilidad y legibilidad son dos términos que delimitan diferente información, aunque a veces se utilizan indistintamente. Un texto puede tener un alto grado de lecturabilidad debido, por ejemplo, al tipo de letra empleado, al color elegido para el fondo, la presencia de gráficas, entre otras, pero resultar ilegible debido a un promedio alto del número de sílabas por palabras, o frases con demasiadas palabras, sin una adecuada estructuración o por una presentación poco clara de sus partes (Kintsch, 1998).

Rubiera, Arbizu, Alzueta, Agúndez y Riera (2004) sugieren como alternativa para mejorar la legibilidad de este tipo de documentos, adaptar este lenguaje técnico con una redacción que combine la complejidad de las frases empleadas, medido bajo el índice de legibilidad integrada, con el de complejidad oracional, ambos de medición automática con procesadores de texto. No obstante, se debe considerar que no siempre es posible modificar este vocabulario técnico pues su alteración por otros términos no siempre es posible (Paasche-Orlow, Taylor y Brancanti, 2003).

Considerando los niveles de legibilidad aceptables para el índice de legibilidad INFLESZ y el de lecturabilidad, según Fernández-Huerta (1959), aproximadamente un $73 \%$ de los consentimientos informados son complejos de leer para una persona y, por tanto, tienen un nivel de comprensión menor. Pero, también los resultados evidencian que un $27 \%$ de los documentos de Consentimiento en el ámbito profesional académico cumplen la función para la que fueron preparados, que es facilitar la información que el investigador presta al participante.

Es conocido que el consentimiento informado es un proceso dialógico, que acompaña y afianza la relación entre médico y paciente o sujeto de estudio y que se refuerza aún más cuando se advierte el esfuerzo profesional de explicar el alcance del estudio o procedimiento para el cual se requiere consentimiento (Ávalos y Tapia, 2013).

Cuando se aplican los documentos de consentimiento informado en ambientes de amplia diversidad, lingüística o cultural o aun cognitiva, se crea con frecuencia la necesidad imperiosa de poner especial atención a la diversidad de capacidades lectoras de los sujetos o pacientes. En todos estos casos, la lecturabilidad y la legibilidad son dos potentes descriptores que facilitan la comprensión de estos documentos.

En el contexto de los discursos especializados del área biomédica, los índices de legibilidad son herramientas de gran utilidad cuando se analizan textos escritos dirigidos a pacientes o sujetos de estudio y son empleados como indicadores de calidad asistencial (Barrio-Cantalejo et al., 2008). El análisis de los diferentes índices de legibilidad o lecturabilidad en la actualidad se realiza con prontitud, precisión y están fácilmente disponibles en los procesadores de texto de uso más habitual, razón por la que el diseño y preparación de los documentos de consentimiento 
informado deberá tender a alcanzar cada vez mayores índices de legibilidad, dada la posibilidad de someter a análisis de rutina a dichos textos.

\section{CONCLUSIONES}

En este artículo se ha obtenido resultados mediante los cuales se puede identificar las estructuras básica y el nivel de legibilidad de los documentos de Consentimiento Informado en el ambito de las ciencias biomédicas.

Analizados 176 (ciento setenta y seis) documentos, los resultados de este estudio demuestran una importante variabilidad para el atributo de legibilidad de los textos analizados. Aproximadamente, un $73 \%$ de los documentos demuestran una baja legibilidad. Esto significa que las frases en el corpus analizado tienden a ser relativamente extensas y con palabras que no son de uso cotidiano, simple. Las estructuras sintácticas no son asimilables fácilmente para los lectores, sujetos de estudio, por lo tanto, tienden a dificultar su comprensión.

A partir de estos resultados se concluye que el formato más frecuentemente usado y el diseño uniforme de estos textos, no garantizan una buena legibilidad de los documentos de Consentimiento Informado.

La cantidad de consentimientos estudiados y la técnica de muestreo sugieren una tendencia que permite generalizar los resultados a las investigaciones científicas tanto en el ámbito académico como en el ámbito profesional biomédico. Un modo de aproximación a un género lingüístico especializado como es el consentimiento informado, es partir desde el supuesto de que los documentos escritos por profesionales del área médica o desde la academia por investigadores de la disciplina, dirigidos a los sujetos participantes de algún tipo de tratamiento o estudio, revelan tanto los tipos de texto, su objetivo y el lenguaje que los sustenta, como rasgos representativos de la disciplina que los genera.

Los documentos escritos desempeñan un papel de apoyo insustituible, siempre y cuando sean confeccionados y usados correctamente. Las funciones básicas de los formularios escritos de consentimiento informado son, en orden de importancia, orientar el proceso de información y de reflexión por parte del sujeto, sobre la medida diagnóstica o terapéutica propuesta entre médico y paciente/sujeto participante de la investigación (proceso que culmina cuando este último autónomamente y sin presiones decide si acepta o rechaza tal medida); servir de registro de que ese proceso se ha llevado a cabo y, por último, facilitar la protección legal del investigador, además de la protección de datos sensibles de parte del sujeto.

A partir de lo anterior, si los documentos de consentimiento informado contienen esta estructura básica y además, se escriben en un lenguaje que posibilite el adecuado entendimiento por parte del sujeto a quien va dirigido, entonces éstos cumplirán cabalmente su rol de comunicar el mensaje desde una parte (tratante o 
investigadora) hacia la parte objeto o sujeto de tratamiento o investigación, condicionando de esta forma el éxito o fracaso de los procedimientos que se describen.

\section{AGRADECIMIENTOS}

Esta investigación está enmarcada en el Proyecto de Tesis para optar al grado de Doctor en Lingüística del Programa de Doctorado en Lingüística de la Universidad de Concepción. Durante el desarrollo del estudio se obtuvo el financiamiento parcial de la Beca Doctorado Nacional de CONICYT y de la Universidad de Concepción.

\section{REFERENCIAS}

Alliende González, Felipe. (2002). La legibilidad de los textos: Manual para evaluación, selección y elaboración de textos. Santiago de Chille, Chile: Andrés Bello.

Anthony, Laurence. (2013). Developing AntConc for a new generation of corpus linguists. Proceedings of the Corpus Linguistics Conference (CL 2013), July 22-26, 2013. Lancaster University, UK, 14-16.

Austin, John Langshaw. (1982). Cómo hacer cosas con palabras. Palabras y acciones. Barcelona, España: Paidós.

Ávalos Nicolás y Tapia Sandra. (2013). Consentimiento informado: síntesis de teoría actual y recomendaciones. Revista chilena de cirugía, 65(5), 448453. Disponible en https://dx.doi.org/10.4067/S0718-40262013000500014.

Ballesteros-Peña, Sendoa y Fernández-Aedo, Irrintzi. (2013). Análisis de la legibilidad lingüística de los prospectos de los medicamentos mediante el índice de Flesch-Szigriszt y la escala Inflesz. Anales del Sistema Sanitario de Navarra, 36(3), 397-406. Disponible en https://recyt.fecyt.es/index.php/ASSN/article/ view/22996.

Barrio-Cantalejo, Inés. (2015). El Programa INFLESZ. Legibilidad.com. Una web sobre el análisis de la legibilidad de textos escritos en español. Disponible en https://legibilidad.blogspot.com/.

Barrio-Cantalejo, Inés y Simón-Lorda, Pablo. (2003). Medición de la legibilidad de textos escritos. Correlación entre método manual de Flesch y métodos informáticos. Atención Primaria. Vol. 31. núm. 2. Febrero 2003, 73-140. Disponible en http://www.elsevier.es/es-revista-atencion-primaria-27-articulo-medicionlegibilidad-textos-escritos-correlacion-13043481.

Barrio-Cantalejo, Inés; Simón-Lorda, Pablo; March Cerdá, Joan y Prieto Rodríguez, María. (2008). Legibilidad gramatical de los prospectos de los medicamentos de más consumo y facturación en España en 2005. Revista Española de Salud Pública, 82(5), 559-566. 
Bhatia, Vijay. (2004). Worlds of written discourse. A genre-based view. London: Continnum.

Biber, Douglas; Connor, Ulla y Upton, Thomas. (2007). Discourse on the Move: Using Corpus Analysis to Describe Discourse Structure. Amsterdam, Netherland: John Benjamins Publishing, 290. ISBN 978-90272-2302-9.

Bonilla-Sandi, Alban. (2003). Consentimiento informado en los experimentos en seres humanos. Medicina Legal de Costa Rica, 20(1), 29-35. Disponible en http://www.scielo.sa.cr/scielo.php?script=sci_arttext\&pid=S140900152003000100004\&lng=en\&tlng=es.

De Beaugrande, Robert y Dressler, Ulrich. (2005). Introducción a la lingüística del texto. Barcelona, España: Ariel.

De la Maza Gazmuri, Ínigo. (2017). Consentimiento informado, un poco de realismo. Revista de Derecho (Valdivia), XXX(2), 111-131.

Emmanuel, Ezekiel. (1999). ¿Qué hace que la investigación clínica sea ética? Siete requisitos básicos. Invesigación en sujetos Humanos: Experiencia Internacional. En A. Pellegrini Filho y R. Macklin. (eds.). Programa Regional de Bioética. División de salud y desarrollo humano (pp. 43-44). Organización Panamericana de la Salud/Organización Mundial de la Salud. Serie Publicaciones.

Fernández-Huerta, José. (1959). Medidas sencillas de lecturabilidad. Consigna, 214, 29-32.

Figueroa, Rodolfo. (2012). Consentimiento informado en la nueva ley de derechos de los pacientes. Revista Médica de Chile, 140(10), 1347-1351.

Hyland, Ken. (2000). Disciplinary discourses. Social interaction in academic writing. Londres: Longman.

Jiménez-Sánchez, Gerardo; Lara, César y Arellano-Méndez, Alberto. (2010). A survey of the development of mexican bioethics: genomic medicine as one of its greatest challenges. En: Pessini Leo, De Barchifontaine Cristian, Lolas Stepke Fernando (Edits). Iberoamerican Bioethics. History and Perspectives. (159-174). Heidelberg, Germany: Springer Dordrecht.

Kintsch, Walter. (1998). Comprehension: A paradigm for cognition. New York, USA: Cambridge University Press.

Kottow, Miguel. (2016). El consentimiento informado en clínica: inquietudes persistentes. Revista Médica de Chile 2016; 144: 1459-1463. Disponible en https://scielo.conicyt.cl/pdf/rmc/v144n11/art12.pdf.

Liguori, Salvatore. (1978). A quantitative assessment of the readability of PPI's. Drug Intelligence and Clinical Pharmacy 1978; 12:712-6. Disponible en http:// journals.sagepub.com/doi/abs/10.1177/106002807801201202?journalCode $=$ aopb.

Lolas Stepke, Fernando. (2010). Bioética en América Latina. Una década de evolución. Centro Interdisciplinario de Estudios en Bioética U. Chile, Monografías 
Acta Bioethica No 4, Centro Interdisciplinario de Estudios en Bioética, Universidad de Chile.

López Calera, Nicolás. (2000). Introducción a los derechos humanos. Granada, España: Comares Editorial.

Ministerio de Salud del Gobierno de Chile. (2006). Ley No 20.120. Sobre investigación científica en el ser humano, su genoma, y prohíbe la clonación humana. Ministerio de Salud del Gobierno de Chile. (2011). Decreto No 114. Aprueba Reglamento de la Ley No 20.120, Sobre la Investigación Científica en el Ser Humano, su Genoma, y Prohíbe la Clonación Humana.

Ministerio de Salud del Gobierno de Chile. (2012). Ley N. 20.584. Regula los Derechos y Deberes que tienen las Personas en Relación con Acciones Vinculadas a su Atención en Salud.

Paasche-Orlow, Michael; Taylor, Holly and Brancanti, Frederick. (2003). Readability standars for informed-consent forms as compared with actual readability. New England Journal of Medicine. 348: 721-726.

Parodi, Giovanni. (2002). El análisis del discurso especializado: aproximación a los tipos de textos utilizados en la formación secundaria técnico-profesional. In I Congreso Internacional de Análisis del Discurso: Lengua, Cultura, Valores.

Parodi, Giovanni. (2003). Relaciones entre lectura y escritura: una perspectiva cognitiva discursiva. Antecedentes teóricos y resultados empiricos. Valparaíso, Chile: Editorial Universitaria de Valparaíso de la Pontificia Universidad Católica de Valparaíso.

Parodi, Giovanni. (2007a). El discurso especializado escrito en el ámbito universitario y profesional: Constitución de un corpus de estudio. Revista Signos, 40(63), 147-178.

Parodi, Giovanni. (Ed.). (2007b). Lingüistica de corpus y discursos especializados: puntos de mira. Ediciones universitarias de Valparaíso. Pontificia Universidad Católica de Valparaíso. Valparaíso, Chile. 461.

Parodi, Giovanni y Ferrari, Silvana. (2007). El discurso de la ciencia y la tecnología en la prensa escrita chilena: aproximación al corpus DICIPE-2004. DELTA: Documentação de Estudos em Lingüistica Teórica e Aplicada, 23(2), 339-369.

Pyrczak, Fred y Roth, Dorothea. (1976). The readability of directions on nonprescription drugs. Journal of the American Pharmaceutical Association, 1976; 16(5): 242-43, 267. Disponible en https:/www.ncbi.nlm.nih.gov/ pubmed/24456020.

Ramírez Durán, María del Valle. (2017). Legibilidad de los consentimientos informados de España y Flandes. Huelva, España: Ed. Universidad de Huelva.

Ramírez-Puerta, Rosario; Fernández-Fernández, Rafael; Frías-Pareja, José; YusteOssorio, María; Narbona-Galdó, Susana y Peñas-Maldonado, Luis. (2013). Análisis de legibilidad de consentimientos informados en cuidados intensivos. Medicina Intensiva, 37(8): 503-509. 
Real Academia de la Lengua Española. (2018). Diccionario de la Lengua Española. Edición del Tricentenario. Disponible en http://dle.rae.es/?id=AP6QLrg. Rubiera, Gerardo; Arbizu, Ricardo; Alzueta, Ángel; Agúndez, Juan y Riera, José. (2004). La legibilidad de los documentos de consentimiento informado en los hospitales de Asturias. Gaceta Sanitaria, 18, 153-158.

Santos, Manuel. (2008). Aspectos bioéticos en los estudios genéticos en poblaciones originarias. En: Elizabeth Lira (Ed.). Bioética en investigación en Ciencias Sociales (115-140). CONICYT Santiago, Chile.

Searle, John. (1986). Actos de habla (L. Valdés Villanueva, trad.), Madrid, España: Cátedra.

Simón-Lorda, Pablo y Concheiro-Carro, Luis. (1993). El consentimiento informado: Teoría y práctica (II) Medicina Clínica Facultad de Medicina de Barcelona, 101: 174-182.

Simón-Lorda, Pablo y Barrio-Cantalejo Inés. (1995). Un marco histórico para una nueva disciplina: la bioética. Medicina Clínica Facultad de Medicina de Barcelona, 105: 583-597.

Swales, John. (2004). Research genres: Explorations and applications. Cambridge: University Press.

UNESCO. Organización de las Naciones Unidas para la Educación, la Ciencia y la Cultura. (2005). Declaración Universal sobre Bioética y Derechos Humanos. 


\section{ANEXO \\ LOGOTIPO \\ CONSENTIMIENTO INFORMADO}

Título del Proyecto: XXXXX

\section{Investigador Responsable: XXXXX}

Se invita a usted a participar del estudio titulado "XXXXX". Antes de que decida si desea participar, o no, es importante que tenga información acerca de este estudio.

Este estudio, que es patrocinado por $\mathbf{X X X X X}$, tiene como objetivo $\mathbf{X X X X X}$.

Conocer si usted está infectado o no XXXXX, permitirá XXXXX.

Si decide participar, usted pasará por los siguientes procedimientos:

1. Inicialmente, $\mathbf{X X X X X}$.

2. Después, $\mathbf{X X X X X}$.

3. Por último, $\mathbf{X X X X X}$.

Participar en este estudio no le generará riesgos ni consecuencias para su salud física.

Es importante señalar que todos los datos personales obtenidos son confidenciales y la información obtenida será utilizada exclusivamente para fines científicos.

Toda la información y los datos serán custodiados por el Investigador Responsable.

Los resultados estarán disponibles para usted, y para ello debe comunicarse con el Investigador Responsable.

Su participación en este estudio, que durará $\mathbf{X X X X X}$, es voluntaria. Si escoge no participar, esto no implica de ningún modo cualquier sanción en razón de su decisión. Si decide participar y cambia de parecer durante el estudio, puede renunciar a su participación en cualquier momento sin sanción de ninguna índole para usted. 
Ante cualquier duda puede realizar las preguntas en cualquier momento de su participación o posteriormente, al correo electrónico del Investigador Responsable, $\mathbf{X X X X X}$. Además, usted puede contactarse con el Comité $\mathbf{X X X X X}$, al teléfono XXXXX.

\section{HOJA DE FIRMAS DOCUMENTO DE CONSENTIMIENTO INFORMADO}

\section{TITULO DEL ESTUDIO: XXXXX}

\section{ANTES DE FIRMAR, CONFIRMO QUE:}

- He sido informado sobre el proyecto de investigación.

- Estoy en conocimiento que la información por mí entregada es confidencial.

- He leído el documento, entiendo las declaraciones contenidas en él y la necesidad de hacer constar mi consentimiento, para lo cual lo firmo voluntariamente, recibiendo en el acto, copia de este documento ya firmado y fechado.

- Mis preguntas han sido respondidas y considero que comprendo toda la información proporcionada acerca del estudio.

- Sé que al firmar este documento, no renuncio a ninguno de mis derechos legales.

\footnotetext{
Nombre del participante

Firma del participante

Fecha de la firma
}

\begin{tabular}{ccc}
\hline $\begin{array}{c}\text { Nombre del Investigador } \\
\text { Responsable }\end{array}$ & $\begin{array}{c}\text { Firma del Investigador } \\
\text { Responsable }\end{array}$ & Fecha \\
& Ren
\end{tabular}

\begin{tabular}{ccc}
\hline Nombre del Director & Firma del Director & Fecha \\
del Centro & del Centro & \\
Ministro de Fe & Ministro de Fe &
\end{tabular}

